\title{
Resting and post exercise Heart Rate Variability in preobese individuals: a comparative study
}

\author{
Rajalingamgari Venkata Mallikarjuna Vara Prasad ${ }^{1}$, Meenakshi Sinha $^{2}$, Jayshri Ghate ${ }^{3}$, \\ Ramanjan Sinha ${ }^{4}$
}

${ }^{1} 3^{\text {rd }}$ Prof MBBS student, All India Institute of Medical Sciences, Raipur (C.G.), ${ }^{2}$ Additional Professor, ${ }^{3}$ Associate

Professor, ${ }^{4}$ Professor and Head, Department of Physiology, All India Institute of Medical Sciences, Raipur (C.G.), India

Background: Several studies have documented high incidence of cardiovascular and metabolic disorders in obese individuals. However, scant and contradictory reports are available in this context for the preobese people, especially in young adults. Aims and Objectives: Therefore, present study was conducted to assess the effect of high BMI (between 24-30) on the time \& frequency domain measures of HRV in normal healthy adult male subjects during resting and post exercise recovery period. Materials and Methods: Healthy young male individuals with no history of any disease were selected and divided in two groups ( $n=15$ in each) on the basis of their BMI: (i) control (BMI 19-24) \& (ii) preobese (BMI 24-30). 15-20 mins of ECG recording was done in the resting state and then till 30 mins after Harvard Step Test. Comparison between groups was done using 2-tailed Mann-Whitney U-nonparametric test. Spearman's Correlation test was used to assess the correlation between BMI and HRV parameters for each group. Results: During resting state, both groups had similar HRV/min. However, control subject had higher parasympathetic tone (high RMSSD, pNN50 \& HF) and low sympathetic tone (low LF \& LF/HF) as compared to preobese individuals. In the post exercise recovery period of control BMI individuals, all the indices of sympathetic measures increased and parasympathetic measures decreased significantly. However, no such changes were observed in preobese group, except significant decrease in the HRV/min. Conclusions: The blunted autonomic response in the post exercise recovery clearly indicates towards a potential risk of cardiovascular compromise in preobese individuals.
Access this article online Website:

http://nepjol.info/index.php/AJMS DOI: 10.3126/ajms.v9i4.20006 E-ISSN: 2091-0576 P-ISSN: $2467-9100$

Key words: Preobese; Body mass index; HRV; Exercise recovery

\section{INTRODUCTION}

Modern life style imposes various challenges to the homeostasis of the body, the major challenges being less physical activity and stressful work environment. These factors lead to disturbed metabolic activity leading to overweight and obesity. Obesity is defined by a body-mass index (BMI) of $30 \mathrm{~kg} / \mathrm{m}^{2}$ or greater. Incidence of obesity in young adults in India now has been identified as one of the biggest precursor of many non-communicable diseases ${ }^{1}$ adversely affecting cardiovascular health of the individual. $^{2}$
The autonomic activity of individuals is known to be the modulator of body weight by its ability to increase energy utilization through activation of sympathetic limb in the situation of higher energy intake. Assessment of ANS activity can help to predict development of cardiovascular diseases in people at risk. ${ }^{3}$ This is often done by objectifying beat-to-beat variation of heart rate from ECG tracing to obtain an idea regarding autonomic influence on myocardial activity. In fact, a reduction in Heart Rate Variability (HRV) has been found to be an authentic mortality predictor in diseases related to the heart, besides also acting similarly in many other diseases. Several reports indicate association 
of high sympathetic and low parasympathetic activity with increased BMI and weight. ${ }^{4,5}$

Some studies have clearly reported absence of any relationship between BMI and heart rate variability (HRV) parameters in pre-obese and obese normotensive adolescents. ${ }^{6,7}$ However, a negative correlation between the waist circumference and RMSSSD \& pNN50 has been reported. ${ }^{6}$ Another study on adolescent group also showed that HRV do not vary during the resting conditions regardless of age and BMI. ${ }^{8}$ But on the other hand, several studies have tried to establish relation between BMI and cardiac autonomic response with change in HRV. Study on adolescent girls reported higher central fat causing higher sympathetic and lower parasympathetic modulations which are indicative of unfavourable HRV indices. ' Another study ${ }^{10}$ not only suggested a relation between BMI and sympathovagal balance, but also that genetic predisposition influences this balance and may be responsible for the inter-individual difference in the susceptibility to gain body weight. Koenig et $\mathrm{al}^{11}$ also reported lower parasympathetic activity with higher BMI; negative correlation of BMI with RMSSD \& pNN50 and suggested that BMI of nonobese, healthy individual is related to the sympathovagal balance. Therefore, these contradictory studies need clearer explanations regarding vagally mediated modulation of metabolism and thereby energy expenditure in human.

Besides, response of obese and overweight people to exercise also show inconclusive results. The overweight and obese people are shown to have suppression of the post-exercise heart rate regulation. ${ }^{12}$ Individuals with higher BMI show longer exercise induced sympathetic activity resulting in decreased HRV with more noticeable change in females. ${ }^{8}$ While, others have suggested delayed return of HRV toward baseline from maximal exercise in people with greater skin fold which was independent of $\mathrm{VO}_{2}$ max and BMI. ${ }^{13}$ Besides, regular physical activity does not only improve fitness but also has positive effect on exercise recovery. In this context, study shows that lower BMI individuals have better exercise recovery. ${ }^{14}$

Thus there exist confusion not only about extent of involvement of sympathetic and parasympathetic activity, but also information about detrimental effect of these changes in people with more modest degrees of overweight (i.e. people with higher BMI but not obese) is lacking with only some studies pointing towards change in sympathovagal balance.

Therefore, the purpose of this study was to assess the changes in HRV measures and thereafter to analyse the degree and nature of relationship between Body Mass Index and HRV indices in resting as well post exercise recovery to the standard Harvard Step Test (HST) in young overweight (preobese) individuals.

\section{MATERIALS AND METHODS}

In this case-controlled prospective experimental study, normal adult male human subjects $(n=30)$ of age 17 to 25 years were included. The study was completely non-invasive with no interventions/ drug administration. Informed consent were obtained from each subject following standard criteria/as per ICMR norms. Ethical Committee permission was also obtained from the host institution before initiating the work and all the procedures followed were in accordance with the ethical standards of the committee and with the Helsinki Declaration of 1975, as revised in 1983. The subjects were divided into two groups on the basis of their BMI: (1) control (BMI between 19-24; $\mathrm{n}=15$ ) and (2) preobese (BMI between 24-30; $n=15)$. A detailed medical history of all the subjects was also taken to meet the inclusion \& exclusion criteria. Any subject with hypertension/diabetes mellitus/respiratory diseases/any other disease history which may affect their autonomic function/history of any neurological or psychological disorder/habits of smoking, alcohol consumption etc. were excluded from the study.

\section{Experimental design}

The study was conducted under the standard laboratory conditions (temperature $26 \pm 2{ }^{\circ} \mathrm{C}$ ) in a single session. After assessment of general health and history of any illnesses, height and weight of the selected subjects was recorded for measurement of BMI $\left(\mathrm{kg} / \mathrm{m}^{2}\right)$ along with their basal heart rate $(\mathrm{HR})$ and respiratory rate.

After a rest for fifteen minutes, resting ECG (for Lead II) recording was done for atleast 20 minutes in supine posture. The ECG signals were obtained with the help of an electronic data acquisition system (Power Lab, AD Instruments Pvt. Ltd., USA) with inbuilt Lab Chart software.

Then, the subjects were asked to perform Harvard Step Test and immediately after the exercise, ECG was recorded for the next 20-30 minutes. However, only the first 5 minutes of recovery ECG data is being presented for the present paper.

\section{Protocol for Harvard step test (HST)}

Subjects were asked to perform standard HST $^{15}$ by alternately step up \& down on a 16" high stool at a rate of 40 steps/minute for a maximum of five minutes or till complete fatigue, whichever occurs earlier. 


\section{Analysis of HRV data}

Artifact free ECG record before and after exercise ( 5 minutes recovery) were selected for the short term HRV analysis from the obtained RR intervals using Lab chart 6 PRO, ADInstruments Pvt. Ltd., USA. In each recording, QRS complexes were detected, and NN (normal to normal) RR intervals were tabulated, yielding an interval tachogram. The variation in the heart rate was then calculated by measuring the difference between minimum and maximum heart rate during inspiration and expiration for the $5 \mathrm{~min}$ period. ${ }^{16,17}$ Then, Power spectral density was determined by Fast Fourier Transformation of interval tachogram. Thereafter, time domain indices i.e., pNN50 (percentage of number of pairs of adjacent RR interval differing by more than $50 \mathrm{msec}$ ) \& RMSSD (square root of the mean squared differences of successive NN intervals) and frequency domain indices i.e., LF (low frequency) power, HF (high frequency) power and total power were obtained. The LF \& HF are expressed in 'normalized unit' (nu) in our study as representation of $\mathrm{LF}$ and $\mathrm{HF}$ in nu emphasizes the controlled and balanced behavior of the two branches of the autonomic nervous system. ${ }^{18}$

\section{Statistical analysis}

Mean \pm SD of HRV parameters for both control and preobese groups in resting and post exercise period were determined. Comparison between groups was done using 2-tailed Mann-Whitney U-nonparametric test. Whereas, Spearman's Correlation test was used to assess the correlation between BMI and HRV parameters for each group. A 'p' value of less than 0.05 was considered statistically significant.

\section{RESULT}

Adult healthy male subjects between the age group of 18-24 years $(\mathrm{n}=30)$ who met all the inclusion \& exclusion criteria were divided into two groups: Group I (control) with BMI between 19-24 and Group II (preobese) with BMI between 24-30.

(i) Physical characteristics: The average BMI of Group I was $21.47 \pm 1.83$ and Group II was $28.25 \pm 1.52$ which was found to be significantly different $(\mathrm{p}<0.001)$.

(ii) Resting/basal condition

(a) Variation in Heart rate variability (HRV/min): No significant difference was detected between the $\mathrm{HRV} / \mathrm{min}$ of control $(25.03 \pm 9.19 / \mathrm{min})$ vs high BMI (24.35 $\pm 9.12 / \mathrm{min})$ individual at resting condition (Fig 1).

(b) HRV indices: The time domain measures, RMSSD \& pNN50 were 67.64 $\pm 30.61 \mathrm{~ms}$ and $35.42 \pm 23.17$ $\%$ respectively for the normal BMI subjects which was significantly higher $(\mathrm{p}<0.05)$ for preobese

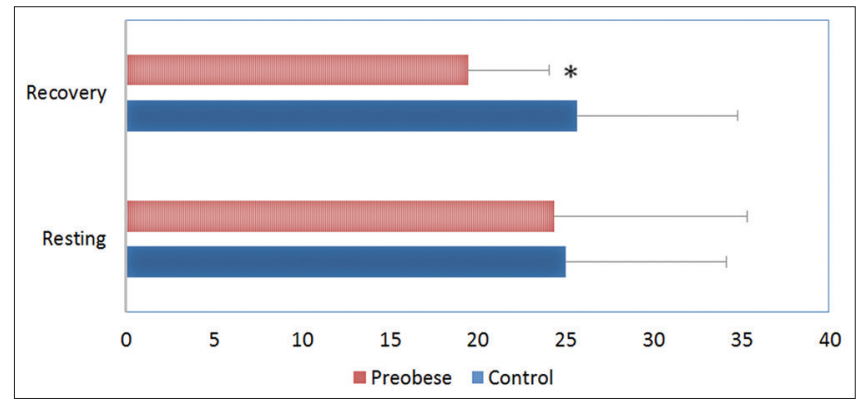

Figure 1 : Depicts comparison of difference of variability of heart rate (HRV/min) during resting and 5 minutes post exercise recovery of subjects with normal (control) and high BMI (preobese). A significant difference was observed between both groups during recovery period. Values are expressed as Mean \pm SD. \# control vs high BMI $p \leq 0.05$

individuals with high BMI (RMSSD 43.85 \pm 23.93 ms and pNN50 19.36 $13.39 \%$ ) (Fig. $2 \mathrm{a} \& 2 \mathrm{~b}$ ). Whereas, among the frequency domain measures, the normal BMI subjects HF (57.85 $\pm 11.45 \mathrm{nu})$ was significantly higher $(\mathrm{p}<0.001)$ than their own $\mathrm{LF}(39.27 \pm 12.88 \mathrm{nu})$ with $\mathrm{LF} / \mathrm{HF}$ ratio of 0.76 . However, no such significant difference existed between $\mathrm{HF}(54.14 \pm 14.57 \mathrm{nu})$ and LF (42.19 \pm 17.2 nu) with LF/HF ratio of 0.95 (Fig.3a, 3b \&3c) for high BMI subjects. No significant difference was detected between the control and preobese group in terms of their HF and LF or LF/HF. However, both $\mathrm{LF}$ and $\mathrm{LF} / \mathrm{HF}$ was higher and $\mathrm{HF}$ was lower in case of high BMI subjects.

(c) Correlation between BMI\& HRV indices: HRV/ min and all other HRV indices (RMSSD, pNN50 $\& \mathrm{HF}$ ) of control subjects were found to have negative correlation with BMI except LF and $\mathrm{LF} / \mathrm{HF}$ which exhibited positive correlation. High BMI subjects also had exactly the same correlation pattern as that of control subjects in resting condition. However, this correlation trend, though consistent, was not found to be statistically significant for any parameters (Table 1).

(iii) Post exercise recovery

(a) Variation in Heart rate variability ( $\mathrm{HRV} / \mathrm{min})$ : A marked decrease in the $\mathrm{HRV} / \mathrm{min}$ was observed for the preobese high BMI group (19.45 $\pm 4.6 / \mathrm{min})$ in the first $5 \mathrm{~min}$ of exercise recovery from their resting value which was significantly low $(p<0.05$, Fig. 1) as compared to recovery HRV of normal BMI subjects (25.69 $\pm 10.98 / \mathrm{min})$. No significant difference was detected between the $\mathrm{HRV} / \mathrm{min}$ of control individual between the resting and recovery conditions.

(b) HRV indices: In the post exercise recovery period, the time domain measures, RMSSD \& pNN50 showed significant $(\mathrm{p}<0.05)$ reduction $(39.73 \pm 27.75 \mathrm{~ms}$ and $6.85 \pm 7.76 \%$ respectively) 


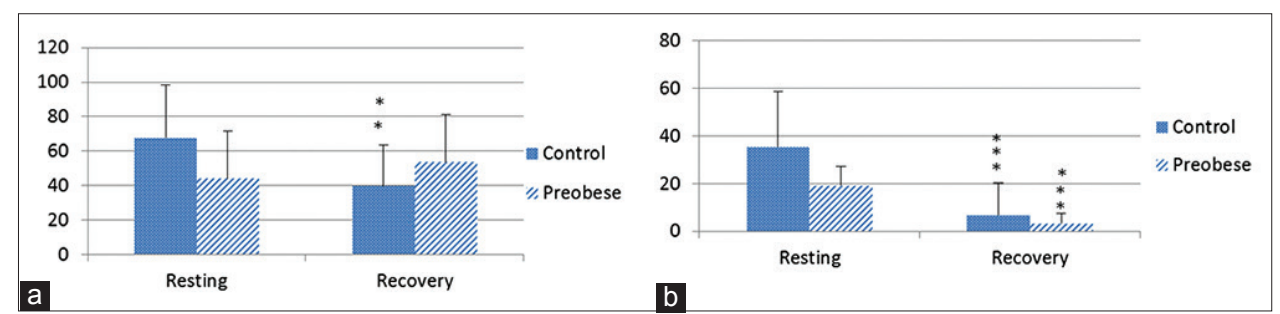

Figure 2 : Change in RMSSD \& pNN50 during resting and 5 minutes post exercise recovery of subjects with normal (control) and high BMI (preobese). (2a) : Significantly reduced RMSSD was seen in preobese group during resting state. Also, RMSSD significantly decreased during recovery stage in the control subjects. (2b) pNN50 decreased significantly in preobese group during resting state. Also, significant reduction in the value was observed during recovery stage in both the groups

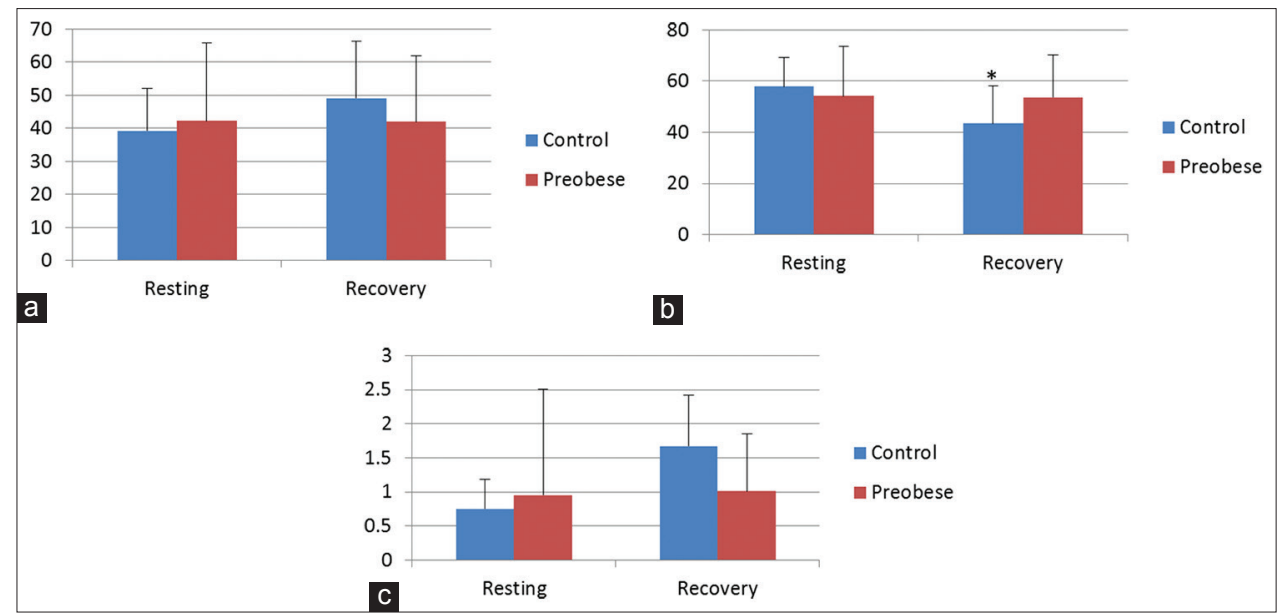

Figure 3 : Shows change in LF, HF \& LF/HF of normal (control) and high BMI (preobese) subjects during resting and 5 minutes post exercise recovery. (3a): Marked increase in LF was observed during recovery stage in the control subjects, though statistically not significant. Preobese subjects did not show any such change. (3b) : Significant fall in HF was noted during recovery of control subjects which was absent in preobese subjects. (3c) : Control subjects had significant increase in their LF/HF ratio during recovery, though statistically not significant. No such change was observed in preobese individuals

\begin{tabular}{|c|c|c|}
\hline & Resting & Post Exercise recovery \\
\hline & BMI & BMI \\
\hline $\mathrm{HRV} / \mathrm{min}$ & NS $(-0.24)$ & $(0.45)^{*}$ \\
\hline pNN50 & NS $(-0.10)$ & NS (0.09) \\
\hline RMSSD & NS $(-0.03)$ & $(0.64)^{\star *}$ \\
\hline LF & NS $(0.17)$ & NS $(-0.41)$ \\
\hline HF & NS $(-0.23)$ & NS (0.29) \\
\hline LF/HF & NS $(0.21)$ & NS $(-0.25)$ \\
\hline
\end{tabular}

for the normal BMI subjects from their resting state. However, RMSSD (53.68 $\pm 27.53 \mathrm{~ms})$ showed increase (though non-significant) and pNN50 (3.44 $3.89 \%)$ showed significant decrease $(p<0.001)$ from their resting state in the high BMI group subjects (Fig 2a \& $2 \mathrm{~b}$ ).

Whereas, in case of frequency domain measures, the normal BMI subjects HF (43.44 $\pm 19.48 \mathrm{nu}$ ) showed significant reduction $(\mathrm{p}<0.05)$ while LF markedly increased $(49.16 \pm 23.73 \mathrm{nu})$ with significant increase in $\mathrm{LF} / \mathrm{HF}$ ratio to $1.67 \pm 1.55$. But, preobese high BMI subjects exhibited no significant difference in $\mathrm{HF}, \mathrm{LF}$ and LF/HF ratio from their resting state (Fig.3a, 3b \&3c). No significant difference was detected between the control and preobese group in terms of their $\mathrm{HF}$ and $\mathrm{LF}$ or $\mathrm{LF} / \mathrm{HF}$.

(c) Correlation between BMI \& HRV indices: Completely opposite correlation pattern emerged for the control subject during recovery in contrast to their resting state i.e. HRV/min, RMSSD \& pNN50 exhibited positive correlation and LF \& LF/HF showed negative correlation with BMI. These correlations were statistically significant for HRV/min \& RMSSD (Table 1). But, preobese with high BMI had no such change in the correlation pattern during recovery with all the pattern (statistically significant for HF) remaining same as that of resting state (Table 2).

\section{DISCUSSION}

Several recent studies have documented that BMI is related to the overall autonomic balance of individuals. However, 


\begin{tabular}{|c|c|c|}
\hline & Resting & Post Exercise recovery \\
\hline & BMI & BMI \\
\hline $\mathrm{HRV} / \mathrm{min}$ & NS $(-0.36)$ & NS $(0.17)$ \\
\hline pNN50 & NS $(-0.44)$ & NS $(-0.30)$ \\
\hline RMSSD & NS $(-0.33)$ & NS $(-0.11)$ \\
\hline LF & NS $(0.32)$ & NS $(0.44)$ \\
\hline $\mathrm{HF}$ & NS $(-0.39)$ & $(-0.47)^{\star}$ \\
\hline LF/HF & NS (0.34) & NS (0.33) \\
\hline
\end{tabular}

the pattern of changes observed in the sympathetic and parasympathetic limb of nervous system due to BMI change is still not clear. Therefore, the present study was an attempt to assess the effect of high BMI (preobese) on otherwise healthy male subjects and also to examine its impact on post exercise recovery in terms of HRV changes.

(i) Physical characteristics: In this study, young adult male subjects were included and divided into two groups i.e. control and preobese (high BMI) group. Both the groups differed significantly in terms of their BMI. As the aim of our study was not to examine the HRV changes in obese individuals, but to assess autonomic changes occurring in pre-obese subjects, subjects having BMI between 24-30 only were included as the experimental group. Obese individuals are known to have cardiovascular and metabolic alterations. Whereas, an apparently healthy pre-obese subject may not have frank changes in his system, but may have latent early changes which, if detected earlier, can be modified with proper lifestyle changes.

(ii) Resting HRV: In our subjects with normal BMI, HRV analysis of all the frequency and time domain measures were comparable to that reported by the Task force group. ${ }^{18}$ Higher parasympathetic/vagal influence was apparent in our subjects because of significantly higher HF (nu) value as compared to LF (Fig $2 \mathrm{a} \& 2 \mathrm{~b}$ ). As is known, HF component $(0.15$ to $0.40 \mathrm{~Hz})$ is indicator of vagal (parasympathetic) activity ${ }^{18}$ as observed during various autonomic challenges eg. electrical stimulation, muscarinic receptor blockade, and vagotomy. Activity in this range is associated with the respiratory sinus arrhythmia, a vagally mediated modulation of heart rate such that it increases during inspiration and decreases during expiration. ${ }^{19}$ Interpreting LF (0.04 to $0.15 \mathrm{~Hz}$ ) component, on the other hand, is controversial. Earlier it was believed to be a marker of sympathetic tone but is now known to include both sympathetic and vagal modulation ${ }^{20}$ and has been associated with baroreflex activity. In the power spectrum, the time domain measures, RMSSD \& pNN50, are closely linked to HF component and therefore, are known to be indicator of parasympathetic activity being linked with cardiac vagal status. Our study corroborates the fact that parasympathetic tone is dominant under resting condition. ${ }^{19}$

Comparison of high BMI (preobese) with normal BMI (control) subjects during resting state, did not have any significant difference in terms of their HRV/min (Fig.1). However, subjects with high BMI, though without any apparent pathology seen in them (all the subjects were strictly selected on the basis of inclusion \& exclusion criteria), had significantly different sympathetic and parasympathetic activity as compared to normal subjects. A lower parasympathetic activity, as evident from HF, RMSD \& pNN50, and high sympathetic tone reflected by $\mathrm{LF} \& \mathrm{LF} / \mathrm{HF}$ in the high BMI subjects clearly suggests for the presence of an early altered state of sympathovagal balance. Besides, even in resting state, the HF and LF components in preobese with high BMI did not differ significantly as was seen in control subjects supporting the earlier statement. Our findings are in accordance to earlier studies ${ }^{11}$. Guizar JM et $\mathrm{al}^{21}$ also have shown in their study that obese adolescents present with reduced parasympathetic and greater sympathetic modulation of cardiac system. In support of sympathovagal balance contributing to regulation of body weight in healthy individuals, Flaa et $\mathrm{al}^{22}$ have suggested sympathoadrenal activity as a negative predictor of future BMI, waist circumference, and triceps skinfold thickness in whites.

Though, we did not find any significant correlation of BMI with HRV indices, both groups of subjects exhibited negative correlation with all the parasympathetic measures (RMSSD, pNN50 \& HF) and positive correlation with sympathetic indicators i.e. LF \& LF/HF (Table 1). This clearly indicates that increasing BMI coincides with reduced parasympathetic and increased sympathetic tone. It may also be reiterated here that high BMI individuals had decreased value of parasympathetic indices and higher sympathetic indices as compared to control subjects. This therefore, justifies the above statement for the correlation pattern.

It has been known that a few individuals maintain a steady weight of the body in spite of overeating, by increasing expenditure of energy proportionally. This type of adaptation which is thermogenic, thereby allowing a few individuals to maintain body weight though overeating is linked to the physiological process called Non-Exercise Activity Thermogenesis (NEAT) which is a mechanism to burn away extra energy. ${ }^{23}$ This activation of NEAT could be due to modulation of the response of autonomic nervous system ${ }^{24}$. Indeed, Christin et $\mathrm{al}^{25}$ reported that there exists variability among different individuals in activity 
of the sympathetic nervous system activity which can be an indicator for the need of frequent body movements by some individuals such as restlessness or fidgeting. On the other hand, an increase in parasympathetic response might slow down the system thus resulting in an increase in deposition of fat. ${ }^{26}$ In comparison, an inversely proportional relationship was observed by A Molfino ${ }^{8}$ between High Frequency (HF) indices and BMI which is agreeable with the data indicating reduction in sympathetic and parasympathetic responses of obese children ${ }^{27}$. Thus it may be possible that an increase in body fat coupled with decrease in parasympathetic activity might indicate a defensive approach by the parasympathetic system against allowing further deposition of fat. Our findings are in similar line as that of A Molfino ${ }^{8} \&$ Nagai et al..$^{27}$ Also, we observed in our study that a trend exists toward increased sympathetic activity in terms of LF \& LF/HF for the subjects with higher BMI (preobese) which was again similar to earlier study. ${ }^{3,10} \mathrm{~A}$ significant correlation of BMI with $\mathrm{LF} / \mathrm{HF}$ ratio and significantly high $\mathrm{LF} / \mathrm{HF}$ ratio has been reported ${ }^{3}$ to be in those with BMI $>25 \mathrm{~kg} / \mathrm{m}^{2}$ as compared to those with BMI $<20 \mathrm{~kg} / \mathrm{m} 2$ and BMI 20 $25 \mathrm{~kg} / \mathrm{m}^{2}$. These facts favor the concept that increasing BMI leads to increased energy expenditure which is modulated by increased sympathetic tone. However, absence of a significant correlation pattern in our study, though very consistent, may be due to smaller sample size (fifteen in each group) and therefore, a larger sample size would reveal clearer correlation pattern.

(iii) Post exercise recovery HRV: A significantly low HRV/ min in the preobese subjects during the first five minutes of exercise recovery as compared to control subject (Fig 1) was very conspicuous in our study. High sympathetic tone and low parasympathetic control is known to represent disturbed autonomic sinus node activity which leads to decrease in HRV/min. ${ }^{3,28}$ Besides, it has been clearly indicated that a reduction in $\mathrm{HRV}$ is a potent marker of cardiovascular diseases. ${ }^{29,30}$ A reduced $\mathrm{HRV} / \mathrm{min}$ in high BMI individuals, as observed in our study therefore, is again a clear indication of early derangement in the sympathovagal balance.

In terms of HRV indices, subjects with normal BMI in our study showed significant reduction in parasympathetic tone as evident by significant reduction in HF, RMMSD \& pNN50. Also, a high sympathetic tone (high LF \& LF/ HF) was observed during the first five minutes of post exercise recovery in these subjects. At this stage, it may be suggested that reduced HRV as observed in them, is expected to be due to fall in vagal tone mainly. This is more so because of the positive correlation of BMI with RMSSD, marker of parasympathetic activity (Table 2). However, no such responses were seen in preobese individuals except a significant fall in pNN50 during recovery (Fig 2a, 2b \& $3 \mathrm{a}, 3 \mathrm{~b}, 3 \mathrm{c})$. In contrast to control subjects, the correlation pattern of high BMI individuals did not show any change from their own resting state. This also may be emphasized here that high BMI individuals did not exhibit any change in their parasympathetic \&/sympathetic indices also (Fig $2 \mathrm{a}, 2 \mathrm{~b} \& 3 \mathrm{a}, 3 \mathrm{~b}, 3 \mathrm{c})$. Sympathetic activation is natural physiological phenomenon during the post exercise period with a fall in parasympathetic tone. However, absence of these responses in high BMI subjects indicate blunting of cardiac autonomic activity. Earlier studies in this regard have also documented suppressed post-exercise heart rate regulation ${ }^{12}$ and reduced $\mathrm{HRV}^{8}$ in the overweight and obese.

In this study, we measured HRV only for 5 min of post exercise recovery and observed that preobese individuals have limited reaction of autonomic nervous system in response to exercise and therefore shows reduced HRV/ min. However, it would be informative and interesting to analyse the recovery pattern of these individuals for longer period i.e. at least for half an hour. The correlation pattern of BMI, though suggestive of definite trend, could not be established conclusively due to non-significant $r$-value (Pearson's R) of many indices. The reason being small sample size, further study with larger number of subjects would help in clarification.

\section{CONCLUSION}

Sedentary lifestyle has become common in the present generation due to fast and competitive world which has led to increased incidence of obesity and many other lifestyles related disorders in the population. The present study clearly indicates an inhibited autonomic response thereby causing reduced HRV in overweight (preobese) individuals in response to even simple and mild to moderate degree of exercise like Harvard Step Test. Basic study conducted on the apparently normal healthy but pre-obese individuals suggest the possible link between BMI and HRV. In fact a 'blunting' of HRV response which was observed when pre- and post-exercise recovery was compared in subjects with high BMI, translates into an alarm for these individuals to adapt to a "autonomic reviving" lifestyle which shall protect them from heart diseases. An early deviation in disturbed sympathovagal balance from the normal, by simply measuring 5 minutes HRV, indicates potential of cardiovascular illnesses. Therefore, this study helps to give early alarm to the youngsters and encourage the individuals with a sedentary lifestyle to take up a more active one which will help in achieving a better cardiovascular fitness and avoid late age cardiovascular diseases. 


\section{ACKNOWLEDGEMENT}

We would like to thank Indian Council of Medical Research (ICMR) for the financial grant sanctioned under Short Term Studentship (STS) fellowship programme (year 2016-17) enabling the work to be carried out successfully.

\section{REFERENCES}

1. Bleich S, Cutler D, Murray $C$ and Adams A. Why is the developed world obese?. Ann Rev Public Health 2008; 29:273-295.

2. Haslam DW and James WP. Obesity. Lancet 2005; 366:11971209.

3. Rajashree $D$ and Vaishali MP. Relationship of body mass index to heart rate variability in young males. Medica Innovatica 2015; 4:10-12.

4. Mohamed FL and Mohamed YS. Relationship of height, weight and BMI to HRV. Sudan Med J 2011, 4:1-11.

5. Balbo SL, Grassiolli S, Ribeiro RA, Bonfleur ML, Gravena C, Brito Mdo N, et al. Fat storage is partially dependent on vagal activity and insulin secretion of hypothalamic obese rat. Endocrine 2007; 31:142-148.

6. Farah $B Q$, Prado WL, Tenório TR and Ritti-Dias RM. Heart rate variability and its relationship with central and general obesity in obese normotensive adolescents. Einstein (Sao Paulo). 2013;11: 285-290.

7. Antelmi I, de Paula RS, Shinzato AR, Peres CA, Mansur AJ and Grupi CJ. Influence of age, gender, body mass index, and functional capacity on heart rate variability in a cohort of subjects without heart disease. Am J Cardiol2004; 93:381-385.

8. Barathi S. Subramaniam. Influence of Body Mass Index on Heart Rate Variability (HRV) in evaluating cardiac function in adolescents of a selected Indian population. Italian $\mathrm{J}$ of Public health $2011 ; 8: 149-155$.

9. Soares M, Alves AJ, Vale S, Aires L, Santos R, Oliveira J, et al. Central fat influences cardiac autonomic function in obese and overweight girls. Pediatr Cardiol 2011;32:924-928.

10. Molfino A, Fiorentini A, Tubani L, Martuscelli M, Rossi Fanelli F and Laviano A. Body mass index is related to autonomic nervous system activity as measured by heart rate variability. Eur J Clin Nut2009; 63: 1263-1265.

11. Koenig J, Jarczok MN, Warth M, Ellis RJ, Bach C, Hillecke TK, et al. Body mass index is related to autonomic nervous system activity as measured by heart rate variability--a replication using short term measurements. J Nutr Health Aging 2014; 18:300-302.

12. Andrew $M$, The effect of BMl on resting heart rate, heart rate variability pre- and post- exercise, and on heart rate regulation post- exercise of $75 \% \mathrm{HRmax}$ in males. The University of Winchester. Thesis for: BSc Dissertation in Sports Science, Advisor: Helen Ryan. April 2014. DOI: 10.13140/ RG.2.2.13005.23525.

13. Michael R Esco and Henry N Williford. Relationship between postexercise heart rate variability and skinfold thickness. Springerplus 2013; 2:389.
14. Föhr T, Pietilä J, Helander E, Myllymäki T, Lindholm H, Rusko H and Kujala UM. Physical activity, body mass index and heart rate variability-based stress and recovery in 16, 275 Finnish employees: a cross-sectional study. BMC Public Health 2016; 16:701-714.

15. Ghai CL. Cardiac efficiency tests: II Harvard step test. In: A textbook of Practical Physiology. 8 ${ }^{\text {th }}$ Ed 2013: $p 186$.

16. Fareedabanu AB, Gorkal AR and Narsimha Shetty KR. A simple test of one minute heart rate variability during deep breathing for evaluation of synpathovagal imbalance in patients with type 2 diabetes mellitus. Pak J Physiol 2011; 7:33-36.

17. Katz A, Liberty IF, Porath A Ovsyshcher I and Prystowsky EN.A simple bedside test of 1 - minute heart rate variability during deep breathing as a prognostic index after myocardial infarction. Am Heart 1999; 138:32-38.

18. Task Force of the European Society of Cardiology and the North American Society of Pacing and Electrophysiology. Heart rate variability: Standards of measurement, physiological interpretation, and clinical use. Circulation 1996; 93:1043-1065.

19. Barett KE, Barman SM, Boitana S and Brooks ML. Cardiovascular regulatory mechanisms. In: Ganong's Review of Medical Physiology, $23^{\text {rd }}$ edition. Lange Publication; 2010:555-568.

20. Billman GE. The LF/HF ratio does not accurately measure cardiac sympatho- vagal balance. Frontiers in Physiology 2013; 4:26.

21. Guízar JM, Ahuatzin R, Amador N, Sánchez G and Romer G. Heart autonomic function in overweight adolescents. Indian Pediat. 2005; 42(5):464-469.

22. Flaa A, Sandvik L, Kjeldsen SE, Eide IK and Rostrup M. Does sympathoadrenal activity predict changes in body fat? An 18-y follow-up study. Am J Clin Nutr2008; 87:1596-1601.

23. Levine JA, Eberhardt NL and Jensen MD. Role of nonexercise activity thermogenesis inresistance to fat gain in humans. Science1999; 283: 212-214.

24. Vanltallie TB. Resistance to weight gain during overfeeding: a NEAT explanation. Nutr Rev 2001; 59:48-51.

25. Balbo SL, Grassiolli S, Ribeiro RA, Bonfleur ML, Gravena C, Brito Mdo N, et al. Fat storage is partially dependent on vagal activity and insulin secretion of hypothalamic obese rat. Endocrine 2007; 31:142-148.

26. Christin L, O'Connell M, Bogardus C, Danforth Jr E and Ravussin E. Norepinephrine turnover and energy expenditure in Pima Indian and white men. Metabolism 1993; 42:723-729.

27. Nagai N, Matsumoto T, Kita $\mathrm{H}$ and Moritani T. Autonomic nervous system activity and the state and development of obesity in Japanese school children. Obes Res 2003; 11:25-32.

28. van Rewanswaaji-Arts CM, Kollee LA, Hopman JC, Stoelinga GB and van Geijn HP. Heart Rate Variability. Ann Intern Med 1993; 118:436-447.

29. Mackay JD, Page MM, Cambridge J and Watkins PJ. Diabetic autonomic neuropathy. The diagnostic value of heart rate monitoring. Diabetologia 1980; 18:471-478.

30. Kliger RE, Miller JP, Bigger JT and Moss AJ. Decreased heart rate variability and its association and its association with increased mortality after acute myocardial infarction. Circulation 1987; 59:256-262. 
Authors Contribution:

P RVMV-Concept, review of literature, collection of data and analysis, preparation of first draft of manuscript; MS-Concept \& design of study, data collection,

statistical analysis \& interpretation, prepared first draft of manuscript \& critical revision of manuscript; JG- Concept \& design of study, data collection, preparation of first draft of manuscript; RS-Conceptualized study, review of literature, statistical analysis \& interpretation, critical revision of manuscript.

Work Attributed to:

Department of Physiology, All India Institute of Medical Sciences, Raipur (C.G.)

Orcid ID:

Mr. RVMV Prasad - http://orcid org/0000-0002-1961-7911

Dr. Meenakshi Sinha - id http://orcid.org/0000-0003-4913-7369

Dr. Jayshri Ghate-10 http://orcid.org/0000-0002-8663-7082

Dr. Ramanjan Sinha- http://orcid.org/0000-0003-1129-5935

Source of Support: Indian Council of Medical Research (ICMR) under Short Term Studentship (STS) fellowship programme (year 2016-17),

Conflict of Interest: None declared. 